
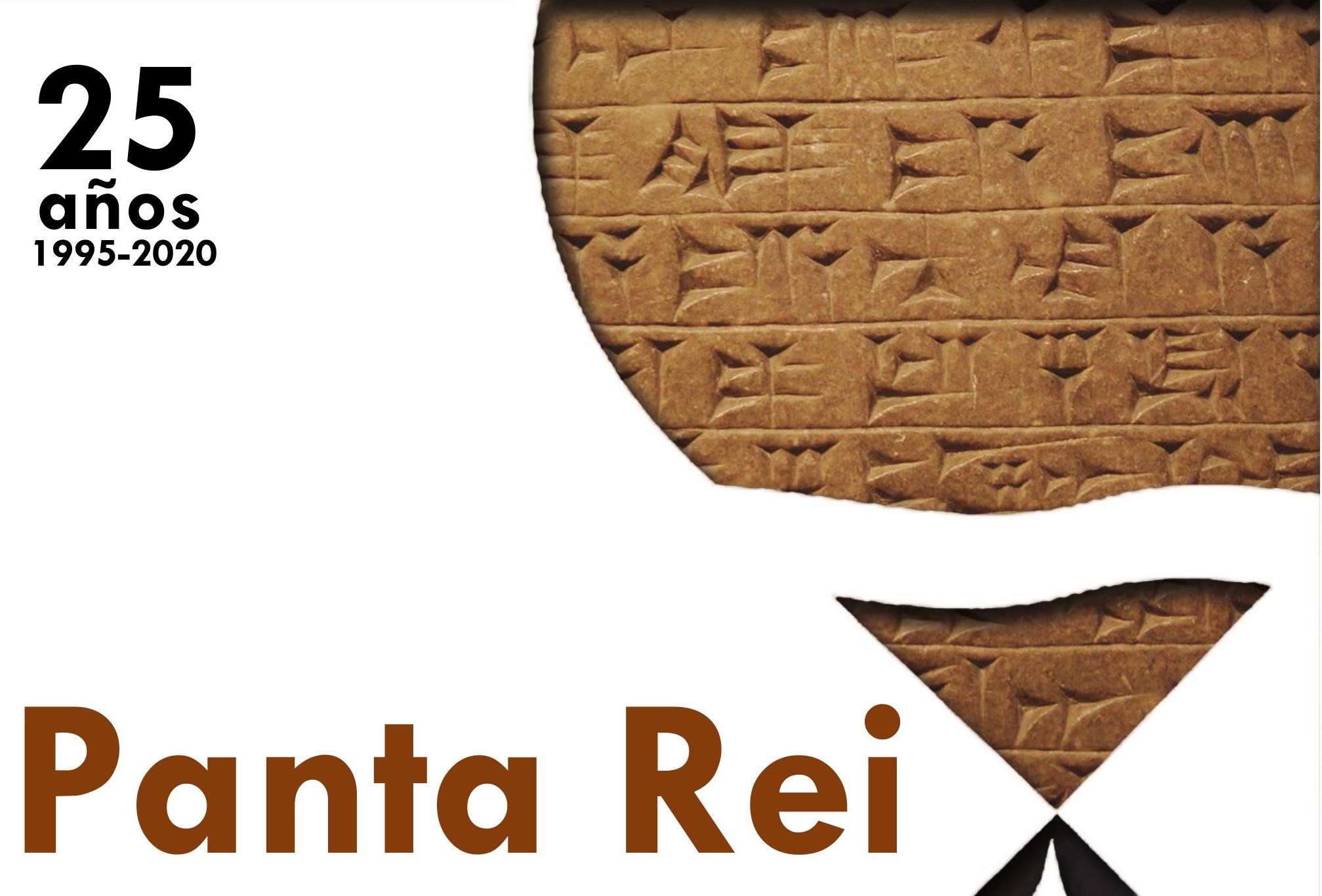

Revista digital de Historia y didáctica de la Historia 


\section{0 (2)}

Revista anual

Fecha de inicio: 1995

Revista Panta Rei. pantarei@um.es

\section{Edita:}

Centro de Estudios del Próximo Oriente y la Antigüedad Tardía - CEPOAT

Edificio Universitario Saavedra Fajardo.

Universidad de Murcia

C/ Actor Isidoro Máiquez, 9

30007 - MURCIA - ESPAÑA

Teléfono: $(+34) 868883890$

cepoat@um.es

Web: www.um.es/cepoat/pantarei

Ediciones de la Universidad de Murcia - EDITUM

Edificio Pleiades. Campus de Espinardo.

Universidad de Murcia

C/ Campus, s/n

30100 - MURCIA - ESPAÑA

Teléfono: $(+34) 868883013$

editum@um.es

Web: https://www.um.es/web/editum/

En portada: Montaje elaborado a partir de fotografía de una inscripción sumeria del British Museum (Wikimedia) (arriba) y fotografía de Miguel Martínez Sánchez (abajo)

Edición 2020 (2)

ISSNe: 2386-8864

ISSN: $1136-2464$
Responsables de los textos: sus autores.

Responsable de la presente edición: Consejo Editorial de Panta Rei.

Depósito legal: MU-966-1995 


\section{CONSEJO DE REDACCIÓN}

Coordinador editorial

Egea Vivancos, Alejandro [Didáctica de las Ciencias Sociales, Universidad de Murcia]

Secretaria

Arias Ferrer, Laura [Didáctica de las Ciencias

Sociales, Universidad de Murcia]

Editores

Jiménez Vialás, Helena [Historia Antigua, Universidad de Murcia]

Martínez Gil, Tània [Didáctica de las Ciencias

Sociales, Universidad de Barcelona]

Meseguer Gil, Antonio José [Historiador, Profesor de

Secundaria]

Ortiz García, Jónatan [Arqueología, Universidad de

Alcalá de Henares]

Romero Molero, Alberto [Arqueología, Universidad

Isabel I]

Sáez Giménez, David Omar [Historiador, Profesor

de Secundaria]

Sáez Rosenkranz, Isidora V. [Didáctica de las

Ciencias Sociales, Universidad de Barcelona]

Sánchez Mondejar, Celso Miguel [Arqueólogo,

Patrimonio Inteligente]

Responsable informático

Martínez García, José Javier [CEPOAT, Universidad de Murcia]

Responsables de traducción y corrección lingüística Martínez Martínez, Cristina [Profesora de Secundaria, Sociedad Española de Lenguas Modernas] Albaladejo Albaladejo, Sara [ISEN-Universidad de Murcia]

\section{CONSEJO ASESOR}

Adroher Auroux, Andrés María [Arqueología, Universidad de Granada]

Albero Muñoz, $M^{a}$ del Mar [H. ${ }^{a}$ del Arte, Universidad de Murcia]

Alia Miranda, Francisco [Historia Contemporánea, UCLM]

Arciniega García, Luis [Historia del Arte, Universidad de Valencia]

Barrio Barrio, Juan Antonio [Historia Medieval,

Universidad de Alicante]

Castellano i Solé, Núria [Egiptología, Schola

Didàctica Activa S.L.]

Chapman, Arthur [History Education, University

College of London, Reino Unido]

Cid López, Rosa María [Historia Antigua, Universidad de Oviedo]

Cobacho López, Ángel [Derecho, Universidad de Murcia]

Cuenca López, José María [Didáctica de las Ciencias Sociales, Universidad de Huelva]
Egea Bruno, Pedro M. ${ }^{a}$ [Historia Contemporánea, Universidad de Murcia]

Feijoo Martínez, Santiago [Arqueología, Consorcio

Ciudad Monumental de Mérida]

García Atienzar, Gabriel [Prehistoria, Universidad de

Alicante]

Ginestí Rosell, Anna [Filología Clásica, Katholische

Universität Eichstätt-Ingolstadt]

González Monfort, Neus [Didáctica de las

Ciencias Sociales, Universidad Autónoma de

Barcelona]

González Soutelo, Silvia [Arqueología, Universidad de Vigo]

Haber Uriarte, María [Prehistoria, Universidad de Murcia]

Hernández de la Fuente, David [Filología Clásica, Universidad Complutense]

Hutson, Scott R. [Anthropology, University of Kentucky, EEUU]

Igual Luis, David [Historia Medieval, UCLM]

Irigoyen López, Antonio [Historia Moderna,

Universidad de Murcia]

Jover Maestre, Francisco Javier [Prehistoria,

Universidad de Alicante]

Mahony, Simon [Digital Humanities, University College of London, Reino Unido]

Marsilla de Pascual, Francisco Reyes [Técnicas

historiográficas, Universidad de Murcia]

Martínez-Burgos García, Palma [H. ${ }^{a}$ del Arte, UCLM]

Mathis, Christian [Didaktik der Geschichte, PH Zürich]

Miralles Maldonado, José Carlos [Filología Clásica,

Universidad de Murcia]

Molina Gómez, José Antonio [Historia Antigua,

Universidad de Murcia]

Mónica Ghirardi [Historia Moderna, Universidad

Nacional de Córdoba, Argentina]

Navarro Espinach, Germán [Historia Medieval,

Universidad de Zaragoza]

Noguera Celdrán, José Miguel [Arqueología,

Universidad de Murcia]

Ortiz Heras, Manuel [Historia Contemporánea, UCLM]

Panzram, Sabine [Historia Antigua, Universität

Hamburg]

Pérez Molina, Miguel Emilio [Filología Clásica,

Universidad de Murcia]

Prados Martínez, Fernando [Arqueología,

Universidad de Alicante]

Sánchez lbáñez, Raquel [Didáctica de las Ciencias

Sociales, Universidad de Murcia]

Sancho Gómez, Miguel Pablo [Educación, UCAM]

Victoria Moreno, Diego [Historia Contemporánea,

UNED]

Vilar García, María José [Historia Contemporánea,

Universidad de Murcia]

Vivas Sainz, Inmaculada [H. ${ }^{a}$ del Arte, UNED]

Zamora López, José Ángel [Próximo Oriente Antiguo,

CCHS-CSIC] 



\section{Índice}

1995-2020. 25 años de Panta Rei

Equipo editorial

La Historia social aplicada a la antigua Mesopotamia: cambios historiográficos y nuevas vías de 9 investigación

Josué Javier Justel Vicente

A Look Back into Ancient Egyptian Linguistic Studies (c. 1995-2019)

Carlos Gracia Zamacona

La infancia en la antigua Mesopotamia: 25 años de investigación

Daniel Justel Vicente

Conflictos armados y su tratamiento en educación. Análisis de la producción científica de los últimos

25 años en la Web of Science

Naiara Vicent, Janire Castrillo, Alex Ibañez-Etxeberria y Leire Albas

La investigación en pensamiento histórico. Un estudio a través de las tesis doctorales de Ciencias

Sociales (1995-2020)

Álvaro Chaparro Sainz, M.a del Mar Felices de la Fuente y Laura Triviño Cabrera

Las narrativas nacionales en la enseñanza y el aprendizaje de la historia: Una revisión sobre los libros de texto y las narrativas de los estudiantes

César López Rodríguez

La historia de las personas: reflexiones desde la historiografía y de la didáctica de las ciencias sociales durante los últimos 25 años

Mariona Massip Sabater, Jordi Castellví Mata y Joan Pagès Blanch †

Panorámica de la trilogía cine, historia y educación en España (1995-2020) 



\title{
La Historia social aplicada a la antigua Mesopotamia: cambios historiográficos y nuevas vías de investigación
}

\section{Social History in Ancient Mesopotamia: Historiographical Shifts and New Venues of Research}

\author{
Josué Javier Justel Vicente \\ Universidad de Alcalá \\ josue.justel@uah.es \\ 0000-0002-8269-0177
}

Recibido: 08/01/2020

Aceptado: 18/04/2020

\begin{abstract}
Resumen
La presente contribución pretende revisar de qué manera la Historia Social, entendida como corriente historiográfica, ha penetrado en el mundo de los estudios cuneiformes y en la Historia de Mesopotamia. De esta manera, se ha realizado una exhaustiva revisión de la bibliografía disponible, sobre todo la referida a los veinticinco últimos años. El resultado principal es que esta tendencia historiográfica ha sido fructífera para el estudio del mundo cuneiforme, si bien dependiendo de periodos y temas ha tenido desigual fortuna. Además, se proporciona una visión (personal) de las líneas de investigación que, en el futuro, podrían seguir desarrollándose en el marco de la Historia Social de la antigua Mesopotamia.
\end{abstract}

\section{Palabras clave}

Estructura social, Historia de Oriente Medio, Historia social, Historiografía, Bibliografía, Historia antigua.

\begin{abstract}
This contribution aims at revising how social history, understood as a historiographical current, has entered the world of cuneiform studies and the history of Mesopotamia. A thorough review of all the available literature has been carried out, especially those studies published during the last twenty-five years. The main outcome is that this historiographical trend has been rather fruitful within the framework of cuneiform studies - yet with unequal results, depending on periods and topics. Finally, a (personal) vision is offered of the areas of research that, in future, may be further developed in the context of the social history of ancient Mesopotamia.
\end{abstract}

\section{Keywords}

Social Structure, Middle Eastern History, Social History, Historiography, Bibliographies, Ancient History.

Para citar este artículo: Justel, J. J. (2020). La Historia social aplicada a la antigua Mesopotamia: cambios historiográficos y nuevas vías de investigación. Panta Rei: revista digital de Historia y didáctica de la Historia, 9-21. doi: 10.6018/pantarei.445361

No sin gran escepticismo llega el historiador, tras su tarea, a aquellos ámbitos intelectuales en los que, de modo sistemático, se habla de órdenes sociales, estructuras y funciones, se manejan estadísticas y se usan símiles o paralelos físico-químicos y aun físico-matemáticos (...). ¿̇Pero hasta qué punto es verdad todo este tinglado? No. La sociedad no es este ente regulador que lo resuelve todo o casi todo con mecanismos sutiles. El historiador (...) sabe que, en realidad, la vida colectiva de los hombres es algo mucho más complejo, contradictorio y dificultoso de limitar de lo que se dice (...) (Caro Baroja, 1970, pp. 281-282). 


\section{Introducción}

Los estudios del Próximo Oriente Antiguo siguen suscitando un enorme interés, tanto entre los profesionales de las disciplinas históricas como entre los aficionados a las mismas. Esto es debido, sobre todo, a que proporcionan puntos de vista muy diferentes de los que nos han venido aportando, tradicionalmente, los estudios del mundo clásico. Este fenómeno es sobre todo visible en ciertos campos que han sido copados casi por completo por la investigación sobre el mundo grecorromano: epigrafía, numismática, economía, administración e instituciones públicas, etc. Por no entrar, por supuesto, en los trabajos de historia puramente política, tema en el que las civilizaciones clásicas han centrado la atención de manera abrumadora. En resumen: la Historia del Próximo Oriente Antiguo en general, y de Mesopotamia en particular, no ha sido objeto de atención preferente por parte de los historiadores de la Antigüedad; y dicho desconocimiento hace, precisamente, que este campo resulte potencialmente más atractivo.

Por su parte, la Historia de Mesopotamia -si bien estudiada marginalmente- tampoco ha quedado descolgada de las grandes tendencias historiográficas en las que nuestra disciplina se ha venido enmarcando. De manera muy general: hubo una primera época en la que primaba más el desarrollo histórico-político de los acontecimientos; siguieron nuevas corrientes historiográficas en las que influyeron otras disciplinas afines -sobre todo procedentes de las ciencias sociales-; $y$, después, un nuevo periodo que vio el renacer de una "nueva" historia política y de las instituciones. Hoy vivimos una renovación de las investigaciones históricas por la influencia de corrientes como el Feminismo, el Posmodernismo, etc. Las obras generales sobre historiografía no contemplan el papel de la Historia de Mesopotamia, y se centran más en las interpretaciones de los historiadores acerca del periodo contemporáneo. Y ello a pesar de que, por las razones indicadas en el párrafo anterior, la Historia de Mesopotamia tiene mucho en qué contribuir a nuestro conocimiento de las interpretaciones del pasado.

\section{Método}

En este artículo pretendo subrayar la tendencia, iniciada durante la segunda mitad del siglo pasado, de estudiar la sociedad "desde abajo"; es decir, lo que se ha venido tradicionalmente adscribiendo a la definición de "Historia Social". Como vamos a ver, esta etapa historiográfica también ha tenido importante presencia en los estudios de la antigua Mesopotamia. Además, dado el carácter de la publicación en que nos encontramos, pretendo destacar especialmente las novedades y cambios que han venido teniendo lugar durante los últimos veinticinco años.

De esta manera, he seguido los siguientes pasos metodológicos:

1) Selección de un amplio listado de títulos sobre el tema.

2) Análisis de las principales tendencias que me ha sido posible descubrir.

3) Integración de todos estos datos en un discurso coherente de las líneas de investigación que más han aportado a la disciplina.

4) Finalmente, identificación de las que son, a mi juicio, las perspectivas más prometedoras para el futuro. 
Se observará, en cualquier caso, que lo que sigue se trata de una selección personal, que se basa en mi amplia experiencia con estos temas y con el mundo cuneiforme; sin duda los lectores podrían estar más interesados en diferentes aspectos del tema. Igualmente, es importante tener en cuenta que he intentado reflejar la bibliografía más reciente, que recogiese los títulos previos (como se indica de manera recurrente en el texto), pero no por ello los más importantes; remito a los lectores interesados a consultar los trabajos citados para ampliar cualquier aspecto.

\section{La Historia Social y Mesopotamia: circunstancias generales}

No es éste el lugar para realizar un análisis de lo que significa la Historia Social ni de otros aspectos relacionados. De ello pueden dar buena cuenta otros autores que se han ocupado en profundidad del análisis historiográfico, si bien casi siempre desde el punto de vista de la Historia Moderna y Contemporánea (Casanova, 1991). Baste decir que la Historia Social es la que se ocupa de estudiar la formación y devenir de las sociedades pasadas, poniendo especial interés en analizar los parámetros de condicionan la vida cotidiana, y prestando atención a los procesos sociales, "por la movilidad ocupacional geográfica y social, tanto entre grupos como entre individuos pertenecientes a ellos, y por las pautas cambiantes de distribución referentes a las tres variables fundamentales: riqueza, poder y estatus" (Stone, 1986, p. 35). Esta definición se ajusta perfectamente a la corriente historiográfica que la investigación sobre la antigua Mesopotamia vivió desde aproximadamente los años sesenta del siglo pasado, y que aún hoy tiene resonancia entre los círculos académicos.

Debe quedar claro, desde el principio, que los cambios historiográficos en Asiriología -como se denomina a la disciplina que estudia los textos de la antigua Mesopotamia- llegan con retraso temporal si los comparamos con otras disciplinas; por ejemplo, respecto a la Historia del mundo clásico (véanse al respecto, por ejemplo, las reflexiones de Bahrani, 2001). Esto es así por la marginalidad de nuestra especialidad, así como el menor número de investigadores dedicados a la misma. Pero quizá también por la exigencia que se ha venido aplicando a estos estudios, donde la misma persona debe ser capaz de leer el soporte cuneiforme, traducir el texto original sumerio o acadio a una lengua moderna, e integrarlo finalmente en sus estudios sobre un tema en cuestión. En cualquier caso: lo que en otras disciplinas fue apareciendo a partir de aproximadamente 1930-1940, en el campo de la Asiriología nos lo encontramos solamente desde 1960, como muy temprano. Es más: en dicha disciplina no tenemos ningún manual general de Historia Social, como sí ocurre con la Roma (Alföldy, 1987) ○ la Grecia antiguas (Gschnitzer, 1987). Es cierto que ha habido intentos en este sentido, pero en general no gozaron de gran aceptación (como es el caso, por ejemplo, de Moscati, 1987), o fueron parciales. En este sentido, un caso paradigmático es el del conocido y excelente volumen de A. Leo Oppenheim, originalmente publicado en $1964\left(2 .^{a}\right.$ ed. de 1977). Se trataba de una aproximación sincrónica y diferente de las que se habían llevado a cabo anteriormente. El libro fue un completo éxito internacional, pero tardó varias décadas en traducirse al español (Oppenheim, 2003).

En el auge de la Historia Social tuvo mucha influencia el progreso y la adaptación de ciencias sociales varias (Casanova, 1996), especialmente la Sociología (véanse especialmente los trabajos de Lloyd, 1988; Noiriel, 1989). Lo mismo ocurrió en la Historia Social aplicada a la antigua Mesopotamia: Economía, Antropología y Sociología tuvieron un enorme impacto en la disciplina de la Asiriología. Como uno de los ejemplos iniciales puede ponerse el estudio de Rivkah Harris (1975) sobre la ciudad de Sippar en época paleobabilónica. Lo que anteriormente habría sido 
un estudio acerca de los principales acontecimientos políticos de la ciudad se transformó, en realidad, en un análisis de los sectores sociales de la ciudad, de sus principales grupos -incluyendo mujeres, esclavos, niños, sacerdotes, etc. - y de las relaciones de poder entre los mismos. Además, el método empleado estaba directamente relacionado con las disciplinas de la demografía histórica y parcialmente de la Cliometría (véase a este respecto, especialmente Williamson, 1990).

Si nos centramos en los cambios de la disciplina durante los últimos veinticinco años, encontramos varias obras que constituyen verdaderos hitos en la Historia Social de Mesopotamia. El primero es sin duda la publicación del manual de Mario Liverani (1995, original italiano de 1988). La obra de Liverani se inserta en el análisis marxista de los acontecimientos y dedica especial atención a las estructuras sociales y socio-económicas, como ha venido haciendo en otras importantes publicaciones, más recientes (Liverani, 2003, 2005). Estos datos los integra en una sucinta contextualización de los hechos políticos, que evidentemente no son lo más relevante del discurso histórico para este autor. Prueba de la importancia de esta obra es el hecho de que se tradujo a múltiples lenguas, y que su última edición ha sido publicada -en inglés, aumentada y corregida- en 2014. A pesar de que ya existían otros manuales generalistas e integraban, con mayor o menor fortuna, aspectos sociales y socio-económicos de Mesopotamia (Avdiev, 1986; Klíma, 1980; y véase la mención arriba del volumen de Oppenheim, 2003 [1964]), la obra de Liverani constituyó un auténtico cambio en el paradigma y, de hecho, se sigue empleando en las facultades de Historia. Un trabajo que podemos considerar similar en aproximación metodológica fue el de Nicholas Postgate (1999), si bien limitado al periodo de ca. 3200-1500 a. C.; otro de idéntica naturaleza, pero que cubre toda la Historia de Egipto antiguo, es el de Trigger, Kemp, O'Connor y Lloyd (1985, con varias ediciones en castellano).

Otro importante hito lo constituye la publicación, en 1995, de una obra que recopilaba de modo coherente artículos de un centenar de autores, de contenido diverso pero que pretendía ofrecer una especie de "manual" que cubriese todo el espectro de la Historia del Próximo Oriente Antiguo (Sasson, 1995). Un buen número de contribuciones estaban dedicadas al análisis de los patrones de poblamiento, la importancia del medio, las instituciones sociales y a factores de economía (partes 2-4 y 6). Aunque los artículos eran dispares tanto por la intención como por otros factores, todos ellos tenían en común un interés directo por los elementos estructurales que rigen la vida de las sociedades del Próximo Oriente Antiguo.

Finalmente se debe destacar otra obra, esta vez de contenido más bien teórico. En 1999 Marc van de Mieroop publicó un ensayo en el que reflexionaba sobre el modo en que la Historia de Mesopotamia había sido contada hasta el momento, y proporcionaba varios ejemplos y temas clave que, en su opinión, merecían mayor atención. De esta manera, diferenciaba entre la historia "desde arriba" (crónicas de los propios reyes, relatos de autoelogio, etc.) y la historia "desde abajo". En esta última el autor incluía la importancia de la prosopografía, de atender a los factores económicos (donde aportaba además una revisión del debate), de la atención que merecían las figuras de las mujeres, etc. Estas reflexiones han resultado ser, de hecho, especialmente visionarias respecto a lo que vendría más adelante, como se va a explicar en el siguiente apartado (§ 4).

No se puede dejar de destacar en esta sección que las obras mencionadas abrieron paso a una multiplicación de estudios, de contenido bastante general, que atendían sobre todo a los 
elementos que arriba se han destacado como definitorios de la "Historia Social". Estas publicaciones, que en algunos casos constituyeron verdaderos best-sellers, sobre todo fueron apareciendo con el cambio de siglo, si bien dejaron de tener notoriedad en la década siguiente (Bertman, 2003; Bottéro, 1996; Nemet-Nejat, 2002; Snell, 1997). No obstante, otros manuales de contenido más general profundizaron en los temas sociales o socio-económicos, e incluso hicieron de los mismos uno de los ejes vertebradores de la historia de Mesopotamia (véase en este sentido, especialmente, Bordreuil, Briquel-Chatonnet y Michel, 2008; exhaustivo pero centrado en época paleobabilónica, por ejemplo Stol, 2004).

\section{La Historia Social y Mesopotamia: algunos temas de interés}

Como se desprende de lo indicado hasta ahora, la Historia Social de Mesopotamia había sido objeto de escasos tratamientos generalistas hasta 1995. Si acaso, desde los años sesenta existían trabajos parciales, centrados en aspectos sociales o económicos muy puntuales, y que no implicaban importantes cambios teóricos. Algunos de estos temas se habían tratado con profundidad ya que, por la propia naturaleza de las fuentes cuneiformes y su antigüedad, resultaban potencialmente de interés para la comunidad académica. Son estos temas los que, durante los últimos veinticinco años, más protagonismo han cobrado. Los aspectos que siguen son los que, a mi juicio, más han destacado.

\subsection{Los grupos sociales}

El de las "clases" y "grupos" sociales ha sido siempre un tema estrella en la historia de Mesopotamia, dado que esta área contiene los registros textuales más antiguos y, por tanto, la historiografía suponía que resolvería varias incertidumbres sobre los primeros estadios de las sociedades estratificadas. Sin embargo, todavía no se ha llegado a entender definitivamente ni éste ni otros aspectos relacionados. Los elementos que se han estudiado desde la perspectiva de la Historia Social son, principalmente, tres: (1) la existencia de grupos sociales bien diferenciados, (2) la naturaleza de la esclavitud en estas sociedades preclásicas, y (3) la importancia de la existencia (o no) de la dicotomía entre el elemento nómada y el sedentario en las sociedades mesopotámicas.

En el primer caso, las discusiones originalmente se centraron, por ejemplo y sobre todo, en las diferencias entre los grupos sociales que aparecían reflejados en el famoso Código de Hammurapi (se observará aquí que hablamos de varones; sobre este tema véase abajo). Desde muy temprano las ciencias sociales se acercaron a este tema y produjeron notables resultados (véanse por ejemplo, con abundantes referencias anteriores, los trabajos de Adams, 1982, 1984; y las interesantes reflexiones metodológicas al respecto de Di Bennardis, 2013). La bibliografía a este respecto resulta apabullante, tanto la general (véase ya Klengel, 1971) como la exclusivamente centrada en época paleobabilónica. En este último caso, el trabajo de Fritz Rudolf Kraus (1958) tuvo gran influencia sobre una multitud de autores, americanos la mayoría, quienes piensan que el término awīlum designaría al hombre libre, mientras que el término muškēnum se refería a un dependiente (véanse por ejemplo Van de Mieroop, 1999; Westbrook, 2003; Fleming, 2004; todos ellos con numerosas referencias previas).

En los últimos años, por su parte, han surgido nuevas ideas acerca de las sociedades próximoorientales, sus divisiones y la movilidad entre sus grupos. $Y$ en este sentido las propuestas de Eva 
von Dassow (2014) contemplan el campo de la Sociología del lenguaje para concluir que, en realidad, los términos awīlum y muškēnum podían ser sinónimos en determinados contextos: el primero se referiría a un hombre que ejerce -en un momento concreto- una autoridad emanada del Estado; el segundo alguien que debe obedecerla. El cambio de perspectiva es, por tanto, no tanto social como administrativo-político.

\subsection{Esclavitud}

Respecto al tema de la esclavitud, es otro de los aspectos estrella de la Historia Social de Mesopotamia. En los últimos años se ha refinado metodológicamente la definición de lo que entendemos por un "esclavo" o una "esclava". De hecho, hay autores que incluso niegan la existencia del fenómeno, entendido a la manera de las sociedades clásicas, pues no siempre es fácil identificar si el trato de individuos se realizó de acuerdo con el derecho de las personas o el derecho de las cosas (véase el planteamiento del debate en Culbertson, 201 1, con bibliografía previa; y abajo para los diferentes grados de servidumbre).

En cualquier caso, conocemos bien la existencia de población servil -esclavos, siervos, o como se quiera denominar- en todas las épocas del Próximo Oriente Antiguo, y sabemos con absoluta certeza que cuando comenzó el registro escrito (ca. 3200 a. C.) ya existía este tipo de personas (Snell, 2011 ). Además, ciertos elementos arqueológicos así lo indicarían también (Porada, Hansen, Dunham y Babcock, 1992). Solamente existe un tratamiento general sobre la esclavitud en Mesopotamia, bastante antiguo y falto de datos (Mendelsohn, 1949); nuevos estudios parciales lo complementarían (Cardellini, 1981; Chirichigno, 1993; Dandamaev, 1984; Molina, 20092011 ; Radner, 2009-2011 ; Snell, 2011 ; Stol, 2009-2011 ; Wilhelm, 2009-2011 ; Wunsch, 20092011, con toda la bibliografía anterior). Estos estudios, más recientes, añaden muchísima información, pero no tratan la misma desde el punto de vista de la Historia Social.

Como se ha anticipado arriba, sabemos que a lo largo de la historia de Mesopotamia hubo diversos grados de esclavitud $y$, por tanto, de libertad. El tema ya había sido planteado con anterioridad, de manera formidable y visionaria, por Ignace J. Gelb $(1973,1979,1982)$ desde Estados Unidos, así como por algunos historiadores del bloque soviético (Korostovtsev, 1977). Ésta ha sido una de las principales líneas de investigación que ha seguido la historiografía en los últimos años. La propia Eva von Dassow (2011) ha reflejado recientemente los últimos avances realizados en este sentido. Lo que queda claro de esta revisión historiográfica es que: (1) había una diferencia entre población libre y esclava; (2) la sociedad era permeable en estos estatus, de manera que una persona podía pasar de una condición a otra; (3) entre la población libre, cada vez más, se fueron diferenciando clases sociales cuya naturaleza parece haber sido, al menos al principio, de carácter militar (Dosch, 2009; Von Dassow, 2008); (4) en muchas épocas de la historia de Mesopotamia se observan grupos de población dependientes, cuyas características e idiosincrasias concretas se nos escapan pero que, en cualquier caso, parecen haber llevado una vida miserable.

En este último sentido, estudios sobre épocas concretas nos han permitido comprobar las penosas condiciones de vida que tenían los trabajadores no cualificados, empleados en explotaciones a gran escala y generalmente dependientes de la administración central (i. e., el Estado). El caso paradigmático, cuyo estudio es muy reciente y se inserta en el campo de la Sociología y de la Antropología, es el de la población dependiente del sur de Mesopotamia durante el Bronce Final 
(Tenney, 2011 a, 2011 b). Incluso se puede ir más allá al hablar de un estatus de semidependencia de sectores de la población -en concreto, de la población judía deportada en Babilonia, ya en época persa-, quienes no eran esclavos, pero estaban controlados de cerca por el poder central (Alstola, 2020).

\subsection{Movilidad geográfica}

Otro aspecto arriba mencionado es el dedicado a la dicotomía entre población sedentaria y nómada. El debate es extensísimo, de lo que da cuenta una publicación de hace una década, generada por un encuentro que trató directamente el tema (Szuchman, 2009, con numerosa bibliografía previa). Los últimos años han estado repletos de publicaciones sobre el tema, la mayoría relacionadas con nuevos textos -o reinterpretación de algunos antiguos- procedentes de los archivos de Mari, en Siria, que datan del s. XVIII a. C. (Durand y Reculeau, 2008, con toda la bibliografía anterior). En esencia, queda claro que la mencionada dicotomía suele ser irreal $\mathrm{O}$, al menos, la población implicada no la entendía como tal. Además, las relaciones entre población sedentaria y nómada eran complejas, y grupos nómadas podían sedentarizarse de manera temporal, y viceversa. En realidad, tanto poblaciones sedentarias como nómadas formaban parte del mismo grupo humano y social, si bien podían tener funciones socio-económicas diferenciadas. Por último, no debe confundirse nomadismo con organización tribal, dado que había poblaciones sedentarias cuya organización social era principalmente tribal (Kärger, 2014).

\subsection{Mujeres y género}

Además de estos temas mencionados, otros que tratan sobre sectores específicos de la sociedad han tenido importancia en los últimos tiempos. Destacaré dos de especial relevancia. El primero de ellos ha cobrado enorme fuerza durante las últimas décadas, y especialmente durante los años más recientes. Se trata de la Historia de las Mujeres en Mesopotamia y, si se quiere ver desde la perspectiva sociológica actual, la Historia del Género. Hasta los años sesenta del siglo pasado apenas había estudios sobre el tema (véase el resumen proporcionado por Bahrani, 2001); desde aproximadamente los años ochenta, los títulos empezaron a multiplicarse, y a día de hoy el listado de trabajos es prácticamente inabarcable (véase el listado bibliográfico completo, más de 500 títulos, en Asher-Greve, 2002 y Garcia-Ventura y Zisa, 2017). En años recientes, además, el número de obras dedicadas al tema y la calidad de las mismas han aumentado exponencialmente, y han ubicado los estudios sobre mujeres mesopotámicas en el contexto, más amplio, de la Historia de las Mujeres en general -y de las del mundo antiguo en particular- (Budin, Cifarelli, Garcia-Ventura y Millet Albà, 2018; Budin y Maclnstosh Turfa, 2016; Chavalas, 2014; Conesa Navarro, Gualda Bernal y Martínez García, 2019; Justel, 2014; Justel y Garcia-Ventura, 2018; Lion y Michel, 2016; Stol, 2016).

No es éste el lugar de repetir los elementos que, a mi juicio, hacen tan importante estudiar el tema de las mujeres en Mesopotamia, además de destacar nuevas vías de investigación (Justel, 2011 ; y cf. Garcia-Ventura, 2016). Baste con destacar que Mesopotamia proporciona la documentación escrita más antigua y en la que, desde el principio, aparecen mujeres en diferentes posiciones sociales y de poder. Un elemento de valor añadido es que estas fuentes nos han llegado tal y como fueron escritas, es decir, no han sufrido modificaciones de autores posteriores, como sí ocurre en época clásica. 


\subsection{Grupos en riesgo de exclusión}

Por último, otro tema que la historiografía ha trabajado, sobre todo recientemente, es el de los grupos sociales en riesgo de exclusión. Uno de ellos, cuyo interés ha crecido enormemente en los últimos años, es el de los niños 0 , mejor dicho, el de la infancia como género. Además, estas contribuciones contextualizan, de nuevo, los casos próximo-orientales entre las informaciones que proporciona el mundo antiguo en general (Flynn, 2019). Trabajos recientes ponen de manifiesto, por ejemplo, desde una perspectiva arqueológica, cómo eran tratados los niños ( $y$, sobre todo, las niñas) a lo largo de varios periodos de la historia del Próximo Oriente Antiguo (Garroway, $2014 a, 2014 b$ ). Otros analizan el papel de la infancia en la conformación de un grupo social que debía ser protegido, sobre todo desde el punto de vista jurídico (D. Justel, 2018, con amplia bibliografía).

Entre estos grupos excluidos y marginados sin duda se encuentran, por ejemplo, las viudas (que suelen ser tratadas en trabajos sobre mujeres o género); pero también personas incapacitadas. La historiografía que estudia la Antigüedad ha procurado tratar este tema en trabajos recientes (Laes, Goodey y Rose, 2013). No es una excepción la disciplina de la Asiriología: por ejemplo, numerosas investigaciones recientes se han centrado en el papel de los invidentes en las sociedades de las que formaban parte (véase J. J. Justel, 2017, con numerosa bibliografía adicional).

\section{Conclusiones y perspectivas de futuro}

De lo anteriormente expuesto se extraen varias ideas claras:

1) La Historia Social de Mesopotamia llegó tarde, si lo comparamos con la historiografía de otros periodos y zonas.

2) No obstante, se ha desarrollado con bastante fluidez, sobre todo durante los últimos veinticinco años, y en temas muy concretos (por ejemplo, el de las mujeres en Mesopotamia).

3) Ha habido un intento, parcialmente logrado, de integrar todos los datos de Mesopotamia en el amplio abanico de fenómenos sociales que tuvieron lugar en otras sociedades del mundo antiguo.

4) No obstante, sigue habiendo temas y tendencias a las que la Historia Social de Mesopotamia no ha llegado, o sus esfuerzos han sido insuficientes.

De esta manera, hay una serie de temas que identificamos en otras disciplinas pero que apenas han sido trabajados para la antigua Mesopotamia. Uno de ellos es el de los movimientos sociales de carácter violento. Si bien no fueron tan frecuentes como en el antiguo Egipto -sobre todo durante época helenística-, sí nos constan fenómenos insurreccionales durante el Primer Milenio a. C. en Mesopotamia. Estos acontecimientos siempre han sido entendidos como movimientos políticos, cuando quizá podrían haber tenido razones sociales. Por ejemplo, falta por estudiar los apoyos populares que recibieron ciertos monarcas que llegaron al poder mediante golpes de Estado.

Otro tema que apenas ha sido trabajado, al menos de manera general, es la posibilidad de ascenso ( $y$ descenso) social. Se han podido estudiar casos muy concretos a lo largo de los tres mil 
años de historia de Mesopotamia: de personajes que mejoraron su estatus social debido a su desempeño de oficios especialmente importantes, entre los que se incluyen a los sacerdotes de templos clave, o de mercaderes que consiguieron mejorar su situación por medio de concesiones - mediante empresas privadas especialmente exitosas. Pero estos casos nunca se han integrado en un estudio coherente, en el que se aplicasen perspectivas sociológicas, y que permitan entender la movilidad vertical en la antigua Mesopotamia.

Finalmente, en mi opinión otro tema tremendamente prometedor es el de la Historia Económica aplicada a los estudios sociales. Hace mucho tiempo que la Historia Económica entró de lleno en los estudios sobre la antigua Mesopotamia (véase el resumen de Van de Mieroop, 1999). Sin embargo, no siempre estos trabajos han tenido como foco de atención la sociedad que produjo el fenómeno. Por ejemplo, los estudios de comportamiento económico de los núcleos semi-urbanos (o semi-rurales) apenas han reflejado el impacto que estos fenómenos tenían en sus correspondientes grupos sociales (los ejemplos de este tipo de estudios son numerosos; véase, con toda la bibliografía anterior, Koliński, 2001). O el conocido debate entre economía palacial y templaria, que está ampliamente superado, no ha reflejado apenas resultados novedosos acerca del impacto de estas investigaciones en la dimensión social del fenómeno (como ejemplo en este caso se puede proponer Prentice, 2010).

\section{Agradecimientos}

Este artículo ha sido posible gracias a una ayuda "Ramón y Cajal" (ref. RYC-2013-13617) concedida por el Ministerio de Economía y Competitividad del Gobierno de España. Cuando las obras indicadas tienen traducción al castellano se ha preferido emplear esta última en las correspondientes referencias.

\section{Bibliografía}

Adams, R. M. (1982). Property Rights and Functional Tenure in Mesopotamian Rural Communities. En M. A. Dandamaev, J. N. Postgate y M. T. Larsen (eds.), Societies and Languages of the Ancient Near East. Studies in Honour of I. M. Diakonoff (pp. 1-14). Warmister: Aris \& Phillips.

Adams, R. M. (1984). Mesopotamian Social Evolution: Old Outlooks, New Goals. En T. Earle (ed.), On the Evolution of Complex Societies: Essays in Honor of Harry Hoijer 1982 (pp. 79-129). Malibu: Undena.

Alföldy, G. (1987). Historia social de Roma. Madrid: Alianza.

Alstola, T. (2020). Judeans in Babylonia. A Study of Deportees in the Sixth and Fifth Centuries BCE. Leiden/Boston: Brill.

Asher-Greve, J. (2002). Women and Gender in Ancient Near Eastern Cultures. Bibliography 1885 to 2001 AD. NIN. Journal of Gender Studies in Antiquity, 3, 33-114.

Avdiev, V. I. (1986). Historia económica y social del Antiguo Oriente. II. Reinos y Estados del II y I milenios. Madrid: Akal.

Bahrani, Z. (2001). Women of Babylon. Gender and Representation in Mesopotamia. London/New York: Routledge.

Bertman, S. (2003). Handbook to Life in Ancient Mesopotamia. New York: Oxford University Press. 
Bordreuil, P., Briquel-Chatonnet, F. y Michel, C. (eds.). (2008). Les débuts de l'histoire. Le ProcheOrient, de l'invention de l'écriture à la naissance du monothéisme. Paris: Éditions de la Martinière.

Bottéro, J. (ed.) (1996). Introducción al antiguo Oriente. De Sumer a la Biblia. Barcelona: Grijalbo Mondadori.

Budin, S. L., Cifarelli, M., Garcia-Ventura, A. y Millet Albà, A. (eds.) (2018). Gender and Methodology in the Ancient Near East. Approaches from Assyriology and Beyond. Barcelona: Universitat de Barcelona.

Budin, S. L. y Maclnstosh Turfa, J. (eds.) (2016). Women in Antiquity. Real Women across the Ancient World. London/New York: Routledge.

Cardellini, I. (1981). Die Biblischen "Sklaven"-Gesetze im Lichte des keilschriftlichen Sklavenrechts. Ein Beitrag zur Tradition, Überlieferung und Redaktion der alttestamentlichen Rechtstexte. Königstein: Hanstein.

Caro Baroja, J. (1970). Inquisición, brujería y criptojudaísmo. Barcelona: Ariel.

Casanova, J. (1991). La Historia Social y los historiadores. ¿Cenicienta o princesa? Barcelona: Crítica.

Casanova, J. (1996). Historia social/sociología histórica: un encuentro sin fusión. Ayer, 22, 105 110.

Chavalas, M. W. (ed.) (2014). Women in the Ancient Near East. London/New York: Routledge.

Chirichigno, G. C. (1993). Debt-Slavery in Israel and the Ancient Near East. Sheffield: Sheffield Academic Press.

Conesa Navarro, P. D., Gualda Bernal, R. M. y Martínez García, J. J. (eds.). (2019). Género y mujeres en el Mediterráneo antiguo. Iconografías y literaturas. Murcia: Universidad de Murcia.

Culbertson, L. (2011). Slaves and Households in the Near East. En L. Culbertson (ed.), Slaves and Households in the Near East (pp. 1-17). Chicago: The Oriental Institute of the University of Chicago.

Dandamaev, M. A. (1984). Slavery in Babylonia from Nabopolassar to Alexander the Great (626331 BC). DeKalb: Northern Illinois University Press.

Di Bennardis, C. (2013). La centralización del poder político y el estado en las sociedades antiguo-orientales: reflexiones sobre teorías e interpretaciones. En C. Di Bennardis, E. Ravenna y I. Milevski (eds.), Diversidad de formaciones políticas en Mesopotamia y el Cercano Oriente (pp. 15-41). Barcelona: Universitat de Barcelona.

Dosch, G. (2009). Zur Struktur der Gesellschaft des Königsreichs Arraphe. Texte über die Streitwagenfahrer (rākib narkabti). Studies on the Civilization and Culture of Nuzi and the Hurrians, 18, 71-228.

Durand, J. M. y Reculeau, H. (2008). Tell Hariri / Mari: Textes. V. Environnement et occupation de l'espace. Supplément au Dictionnaire de la Bible, 14, 298-356.

Fleming, D. E. (2004). Democracy's Ancient Ancestors: Mari and Early Collective Governance. Cambridge: Cambridge University Press. 
Flynn, S. W. (ed.) (2019). Children in the Bible and the Ancient World. Comparative and Historical Methods in Reading Ancient Children. London/New York: Routledge.

Garcia-Ventura, A. (2016). Investigación feminista, Historia de las Mujeres y mujeres en los estudios sobre Próximo Oriente Antiguo. Revista de Estudos Culturais, 3. Recuperado de http://www.revistas.usp.br/revistaec/article/view/1 49562, último acceso 31/12/2019.

Garcia-Ventura, A. y Zisa, G. (2017). Gender and Women in Ancient Near Eastern Studies: Bibliography 2002-2016. Akkadica, 138, 37-67.

Garroway, K. H. (2014a). Children in the Ancient Near Eastern Household. Winona Lake: Eisenbrauns.

Garroway, K. H. (2014b). Neither Slave Nor Free: Children Living on the Edge of a Social Status. En B. T. Arnold, N. L. Erickson y J. H. Walton (eds.), Windows to the Ancient World of the Hebrew Bible. Essays in Honor of Samuel Greengus (pp. 121 -137). Winona Lake: Eisenbrauns.

Gelb, I. J. (1973). Prisoners of War in Ancient Mesopotamia. Journal of Near Eastern Studies, 32, 70-98.

Gelb, I. J. (1979). Definition and Discussion of Slavery and Freedom. Ugarit-Forschungen, 11 , 283297.

Gelb, I. J. (1982). Terms for Slaves in Ancient Mesopotamia. En M. A. Dandamaev, J. N. Postgate y M. T. Larsen (eds.), Societies and Languages of the Ancient Near East. Studies in Honour of I. M. Diakonoff (pp. 81-98). Warmister: Aris \& Phillips.

Gschnitzer, F. (1987). Historia social de Grecia. Desde el Periodo Micénico hasta el final de la Época Clásica. Madrid: Akal.

Harris, R. (1975). Ancient Sippar. A Demographic Study of an Old-Babylonian City (1894-1595 B.C.). Istanbul: Nederlands Historisch-Archaeologisch Instituut te Istanbul.

Justel, D. (2017). Infancia y legalidad en el Próximo Oriente antiguo durante el Bronce Reciente (ca. 1500-1 100 a. C.). Atlanta: Society of Biblical Literature.

Justel, J. J. (2011). Mujeres y género en la historiografía del Próximo Oriente Antiguo: pasado, presente y futuro de la investigación. Arenal. Revista de Historia de las mujeres, 18, 371 407.

Justel, J. J. (2014). Mujeres y derecho en el Próximo Oriente Antiguo. La presencia de mujeres en los textos jurídicos cuneiformes del segundo y primer milenios a. C. Zaragoza: Pórtico.

Justel, J. J. (2017). Blindness in Nuzi Texts. Die Welt des Orients, 47, 242-258.

Justel, J. J. y Garcia-Ventura, A. (eds.) (2018). Las mujeres en el Oriente cuneiforme. Alcalá de Henares: Universidad de Alcalá.

Kärger, B. (2014). Leben in der amurritischen Welt. Nomaden und Sesshafte im Reich von Mari im 19. und 18. Jahrhundert vor Christus. Wiesbaden: Harrassowitz.

Klengel, H. (ed.) (1971). Beiträge zur sozialen Struktur des alten Vorderasien. Berlin: Akademie Verlag.

Klíma, J. (1980). Sociedad y cultura en la antigua Mesopotamia. Madrid: Akal.

Koliński, R. (2001). Mesopotamian dimātu of the Second Millennium BC. Oxford: Archaeopress. 
Korostovtsev, M. A. (1977). Was ist ein Sklave? Altorientalische Forschungen, 5, 5-15.

Kraus, F. R. (1958). Ein Edikt des Königs Ammi-saduqa von Babylon. Leiden: Brill.

Laes, C., Goodey, C. F. y Rose, M. L. (eds.) (2013). Dissabilities in Roman Antiquity: Disparate Bodies A Capite ad Calcem. Leiden/Boston: Brill.

Lion, B., y Michel, C. (eds.). (2016). The Role of Women in Work and Society in the Ancient Near East. Boston/Berlin: Walter de Gruyter.

Liverani, M. (1995). El antiguo Oriente. Historia, sociedad y economía. Barcelona: Crítica.

Liverani, M. (2003). Relaciones internacionales en el Próximo Oriente Antiguo, 1600-1100 a.C. Barcelona: Bellaterra.

Liverani, M. (2005). Más allá de la Biblia. Historia antigua de Israel. Barcelona: Crítica.

Lloyd, C. (1988). Explanation in Social History. Oxford/New York: Basil Blackwell.

Mendelsohn, I. (1949). Slavery in the Ancient Near East: A Comparative Study of Slavery in Babylonia, Assyria, Syria, and Palestine from the Middle of the Third Millenium to the End of the First Millenium. New York: Oxford University Press.

Molina, M. (2009-201 1). Sklave, Sklaverei. A. Im 3. Jahrtausend. Reallexikon der Assyriologie und Vorderasiatischen Archäologie, 12, 562-564.

Moscati, S. (ed.) (1987). El alba de la civilización. Sociedad, economía y pensamiento en el Próximo Oriente Antiguo. Tomo I: La sociedad. Madrid: Ediciones Cristiandad.

Nemet-Nejat, K. R. (2002). Daily Life in Ancient Mesopotamia. Peabody: Greenwood.

Noiriel, G. (1989). Pour une approche subjectiviste du social. Annales. Économies, sociétés, civilisations, 6, 1435-1459.

Oppenheim, A. L. (2003). La antigua Mesopotamia. Retrato de una civilización extinguida (edición ampliada por Erica Reiner). Madrid: Gredos.

Porada, E., Hansen, D., Dunham, S. y Babcock, S. (1992). The Chronology of Mesopotamia ca. 7000-1600 BC. En R. Ehrich (ed.), Chronologies in Old World Archaeology: Third Edition (pp. 77-121). Chicago: The University of Chicago Press.

Postgate, J. N. (1999). La Mesopotamia arcaica. Sociedad y economía en el amanecer de la historia. Madrid: Akal.

Prentice, R. (2010). The Exchange of Goods and Services in Pre-Sargonic Lagash. Münster: UgaritVerlag.

Radner, K. (2009-2011). Sklave, Sklaverei. C. Neuassyrisch. Reallexikon der Assyriologie und Vorderasiatischen Archäologie, 12, 571-572.

Sasson, J. M. (ed.) (1995). Civilizations of the Ancient Near East. New York: Charles Scribner's Sons.

Snell, D. C. (1997). Life in the Ancient Near East. New Haven: Yale University Press.

Snell, D. C. (2011). Slavery in the Ancient Near East. En K. Bradley y P. Cartledge (eds.), The Cambridge World History of Slavery. Volume 1: The Ancient Mediterranean World (pp. 421). Cambridge: Cambridge University Press. 
Stol, M. (2004). Wirtschaft und Gesellschaft in altbabylonischer Zeit. En D. Charpin, D. O. Edzard y M. Stol (eds.), Mesopotamien. Die altbabylonische Zeit. Fribourg/Göttingen: Vandenhoeck \& Ruprecht.

Stol, M. (2009-2011). Sklave, Sklaverei. B. Altbabylonisch. Reallexikon der Assyriologie und Vorderasiatischen Archä̈logie, 12, 564-571.

Stol, M. (2016). Women in the Ancient Near East. Boston/Berlin: Walter de Gruyter.

Stone, L. (1986). El pasado y el presente. Mexico: Fondo de Cultura Económica.

Szuchman, J. (ed.) (2009). Nomads, Tribes, and the State in the Ancient Near East. Cross-Disciplinary Perspectives. Chicago: The Oriental Institute of the University of Chicago.

Tenney, J. S. (2011 1a). Household Structure and Population Dynamics in the Middle Babylonian Provincial "Slave" Population. En L. Cutbertson (ed.), Slaves and Households in the Near East (pp. 135-146). Chicago: The Oriental Institute of the University of Chicago.

Tenney, J. S. (201 1b). Life at the Bottom of Babylonian Society: Servile Laborers at Nippur in the 14th and 13th Centuries B.C. Leiden/Boston: Brill.

Trigger, B. G., Kemp, B. J., O'Connor, D. y Lloyd, A. B. (1985). Historia del Egipto Antiguo. Barcelona: Crítica.

Van de Mieroop, M. (1999). Cuneiform Texts and the Writing of History. London/New York: Routledge.

Van de Mieroop, M. (1999). The Government of an Ancient Mesopotamian City. En K. Watanabe (ed.), Priests and Officials in the Ancient Near East (pp. 139-161). Heidelberg: Universitätsverlag C. Winter.

Von Dassow, E. (2008). State and Society in the Late Bronze Age. Alalah under the Mittani Empire. Bethesda: CDL Press.

Von Dassow, E. (201 1). Freedom in Ancient Near Eastern Societies. En K. Radner y E. Robson (eds.), The Oxford Handbook of Cuneiform Culture (pp. 205-224). Oxford: Oxford University Press.

Von Dassow, E. (2014). Awīlum and Muškēnum in the Age of Hammurabi. En L. Marti (ed.), La famille dans le Proche-Orient ancien: réalités, symbolismes, et images (pp. 291-308). Winona Lake: Eisenbrauns.

Westbrook, R. (2003). Old Babylonian Period. En R. Westbrook (ed.), A History of Ancient Near Eastern Law (pp. 361-430). Leiden/Boston: Brill.

Wilhelm, G. (2009-2011). Sklave, Sklaverei. C. Bei den Hethitern. Reallexikon der Assyriologie und Vorderasiatischen Archäologie, 12, 574-576.

Williamson, J. G. (1990). La Cliometría: una visión norteamericana. Revista de Historia Económica - Journal of Iberian and Latin American Economic History, 8, 39-52.

Wunsch, C. (2009-2011). Sklave, Sklaverei. D. Neubabylonisch. Reallexikon der Assyriologie und Vorderasiatischen Archä̈logie, 12, 572-574. 


\section{Panta Rei}

PANTA REl es una revista digital de investigación orientada a la Historia y la Didáctica de la Historia. Su principal objetivo es la transmisión del conocimiento científico, dando una oportunidad también a los jóvenes investigadores que quieren abrirse camino en el estudio de las ciencias humanas y sociales. Se compone de estudios originales relacionados con la disciplina histórica así como su didáctica y difusión. Las diferentes secciones que componen la revista son: artículos de investigación, entrevistas a profesionales, recensiones de monografías de actualidad y crónicas de congresos o eventos científicos relevantes.

Todos los artículos publicados son objeto de un proceso de revisión a cargo de un mínimo de dos evaluadores, que se consideran expertos en el ámbito temático del artículo propuesto. Nuestro deseo es poder ofrecer unos contenidos rigurosos, de calidad y de interés.

El CEPOAT (Centro de Estudios del Próximo Oriente y la Antigüedad Tardía de la Universidad de Murcia) es la institución encargada de la coordinación y gestión de la revista, desde donde anualmente se lanzará la convocatoria para aquellos que estén interesados en publicar sus trabajos, siempre relacionados con la Historia y la Didáctica de la Historia.

PANTA REI is a digital journal focused on History and Teaching History. Its main objective is the transmission of scientific knowledge by giving also an opportunity to young researchers who want to make their way in the study of human and social sciences. It is composed by original studies related to History, as well as its didactics and promotion. The different sections of this journal are: research articles, interviews to professionals, recensions on monographs about current issues and reports about congresses or relevant scientific events.

All the articles published are subject to a revision process carried out by a minimum of two reviewers who are considered to be experts in the field of the article proposed. Our wish is to offer rigorous contents with quality and being of interest to the reader.

CEPOAT (Centre of Studies of the Middle East and Late Antiquity of the University of Murcia) is the institution in charge of the coordination and management of this journal. This is the centre from where the call for papers will be launched annually for all the people interested in publishing their papers, always related to History and Teaching History. 


\section{Normas de publicación}

El autor se compromete a enviar trabajos originales, que no se encuentren publicados en otras revistas ni en otros idiomas. Así mismo, el mismo artículo no podrá ser presentado en otras revistas mientras dure el proceso de evaluación.

\section{Envío y presentación de originales}

Las normas de edición y forma de envío de artículos a la revista se pueden consultar en https://revistas.um.es/pantarei/

Para la redacción de los trabajos se tendrá en cuenta el Manual de la American Psychological Association, en su $7 .^{a}$ edición. La extensión máxima de los trabajos será de 25 páginas. La revista acepta originales escritos en español o inglés.

\section{Proceso de valoración y evaluación}

Una vez recibidos los trabajos, la Revista realizará una primera valoración. Si el trabajo enviado se ajusta a las normas de presentación propuestas, la temática es coincidente con la línea editorial de la revista y posee la calidad científica necesaria, será remitido al consejo asesor para una primera evaluación. Si no es así en este primer paso se puede rechazar directamente los documentos que incumplan claramente la línea editorial.

Será el Consejo Asesor quien indique a la revista la originalidad, relevancia, estructura, redacción, aparato bibliográfico, etc. del trabajo enviado y, para ello, se designará a dos revisores expertos externos que evaluarán cada uno de los trabajos, que pueden formar parte (o no) de este Consejo Asesor. La selección de los revisores se ajustará a la temática y características metodológicas del trabajo. El nombre y filiación de los autores serán eliminados del trabajo para su revisión, así como los revisores actuarán de manera anónima y confidencial.

Los revisores deberán rellenar un informe de evaluación que centrará su atención en aspectos tales como características formales, originalidad y novedad de los trabajos, relevancia de las propuestas y los resultados, calidad metodológica y validez científica.

Una vez terminado el proceso se decidirá la aceptación o no de los mismos y su publicación en el número que sea pertinente, así como las modificaciones susceptibles de ser realizadas para su final publicación. Dicha notificación se enviará únicamente por correo electrónico, en un plazo máximo de seis meses. 


\section{Publishing rules}

The author is committed to submit original papers not having been published in other reviews or in other languages. In this way, it is not allowed for the same paper to be presented in other reviews during the evaluation process.

\section{Submission and presentation of originals}

The editing rules and the guidelines for the submission of papers can be consulted at https://revistas.um.es/pantarei/

The seventh edition of the Manual of the American Psychological Association will be taken into account for the writing of the papers. The length of the submitted papers will not exceed the 25 pages. The journal accepts originals written in Spanish or English.

\section{Examination and assessment process}

The Journal will submit the papers to a first examination once received. If the paper follows the presentation guidelines, the subject agrees with the editorial line of this journal, and possess the scientific quality required, it will be sent to the advisory council for a first assessment. If not, the documents which clearly fail to complete the editorial line may be rejected straightaway in this first step.

The Advisory Council will indicate the originality, relevance, structure, writing, bibliography, etc. of the text to the journal; for this purpose, two outside experts will be designated to review the papers; these experts can be (or not) part of this Advisory Council. The selection of the experts will adjust to the subject and methodological characteristics of the paper. Name and affiliation of the author will be eliminated from the text for its review, in this way experts will act anonymously and confidentially.

The experts will fill out an assessment report which will focus on aspects such as formal characteristics, originality and novelty of the papers, relevance and results of the proposal, methodological quality and scientific validity.

Once the process is finished, the acceptance or not of the papers and its publication in the corresponding edition will be decided, as well as the modifications that may be done for its final publication. This notification will be sent by email within 6 months maximum. 


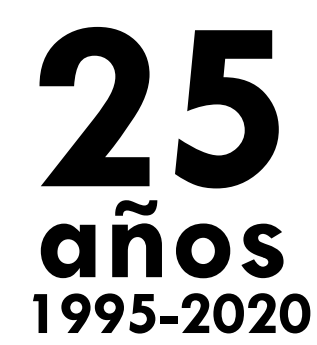

\section{cepoAt editum}

